\title{
Some Variants of Vaught's Conjecture from the Perspective of Algebraic Logic
}

\author{
Gábor Sági* and Dorottya Sziráki
}

November 25, 2011

\begin{abstract}
Vaught's Conjecture states that if $\Sigma$ is a complete first order theory in a countable language such that $\Sigma$ has uncountably many pairwise non-isomorphic countably infinite models, then $\Sigma$ has $2^{\aleph_{0}}$ many pairwise non-isomorphic countably infinite models.

Continuing investigations initiated in [17], we apply methods of algebraic logic to study some variants of Vaught's conjecture. More concretely, let $S \subseteq{ }^{\omega} \omega$ be a $\sigma$-compact monoid. We prove, among other things, that if a complete first order theory $\Sigma$ has at least $\aleph_{1}$ many countable models which cannot be elementarily embedded into each other by elements of $S$, then, in fact, $\Sigma$ has continuum many such models. We also study related questions in the context of equality free logics and obtain similar results.

Our proofs are based on the representation theory of cylindric and quasipolyadic algebras (for details see [9] and [10]) and topological properties of the Stone spaces of these algebras.
\end{abstract}

AMS Subject Classification: Primary 03C45, 03G15; Secondary 03C30. Keywords: Vaught's conjecture, countable models, elementary embeddings, equality free logics, quasi-polyadic algebras, representations.

\section{Introduction}

Let $\Sigma$ be a complete first order theory in a countable language. Recall that for any cardinal $\kappa, I(\Sigma, \kappa)$ denotes the number of pairwise non-isomorphic models of $\Sigma$ of cardinality $\kappa$. The following statement has become known as Vaught's conjecture: if, counting up to isomorphism, $\Sigma$ has at least $\aleph_{1}$ many countable models, then $\Sigma$ has $2^{\aleph_{0}}$ many countable models; in symbols:

*Supported by Hungarian National Foundation for Scientific Research grant K68262 and by the János Bolyai Research Scholarship of the Hungarian Academy of Sciences. 


$$
(*) \quad I\left(\Sigma, \aleph_{0}\right)>\aleph_{0} \text { implies } I\left(\Sigma, \aleph_{0}\right)=2^{\aleph_{0}} \text {. }
$$

This conjecture has become an important open problem, and was mentioned in practically all monographs on model theory. See, for example Hodges [11], on page 339, (and Shelah [22], Chang-Keisler [6], Buechler [5], and Marker [12]). The original conjecture was published in Vaught [23].

Vaught's conjecture stimulated an intensive research; we recall the following related results:

- Morley proved in [13] that $I\left(\Sigma, \aleph_{0}\right)>\aleph_{1}$ implies $I\left(\Sigma, \aleph_{0}\right)=2^{\aleph_{0}}$ (see also Theorem 4.4.16 of [12]);

- Bouscaren and Lascar proved in [3] that $(*)$ is true for $\aleph_{0}$-stable $\Sigma$ of finite Morley rank;

- Shelah proved in $[21]$ that $(*)$ is true for $\aleph_{0}$-stable $\Sigma$ and

- Buechler proved in [4] that $(*)$ is true for superstable $\Sigma$ of finite $U$-rank.

In the last three results above, Vaught's conjecture was proven for theories satisfying certain extra conditions. These conditions originate from stability theory. In the present work, we follow another approach; (particularly, we do not deal with stability theory).

Section 4 of [17] suggests two intriguing research directions. First off, one could study Vaught's conjecture in first order logic without equality. Secondly, instead of considering models up to isomorphism as in the conjecture, one could consider models up to elementary embeddability. Thus we have Problems 1-4 below: the original conjecture, and three possible variants. Here, $I^{\prime}(\Sigma, \kappa)$ denotes the supremum of cardinalities of sets containing pairwise non elementarily embeddable $\kappa$-sized models of $\Sigma$. We note that in the case of languages without equality, an elementary mapping, (ie. a fuction that preserves all $\emptyset$-definable relations), is not necessarily injective. However, throughout the paper, an elementary embedding is defined as an injective elementary mapping, (and so elementary embeddings are injective in the equalityfree case too). We refer to the fourth paragraph of Section 6 in connection with this issue.

Open problem 1.1 (What has become known as Vaught's Conjecture) Let $\Sigma$ be a complete theory in a countable language with equality. Is it true that if $I\left(\Sigma, \aleph_{0}\right)>\aleph_{0}$, then $I\left(\Sigma, \aleph_{0}\right)=2^{\aleph_{0}}$ ?

Open problem 1.2 Let $\Sigma$ be a complete theory in a countable language without equality. Is it true that if $I\left(\Sigma, \aleph_{0}\right)>\aleph_{0}$, then $I\left(\Sigma, \aleph_{0}\right)=2^{\aleph_{0}}$ ?

Open problem 1.3 Let $\Sigma$ be a complete theory in a countable language with equality. Is it true that if $I^{\prime}\left(\Sigma, \aleph_{0}\right)>\aleph_{0}$, then $I^{\prime}\left(\Sigma, \aleph_{0}\right)=2^{\aleph_{0}}$ ? 
Open problem 1.4 Let $\Sigma$ be a complete theory in a countable language without equality. Is it true that if $I^{\prime}\left(\Sigma, \aleph_{0}\right)>\aleph_{0}$, then $I^{\prime}\left(\Sigma, \aleph_{0}\right)=2^{\aleph_{0}}$ ?

Since we are dealing with countable models, we may assume, without loss of generality, that the universe of these models is always a fixed countably infinite set $\omega$. More formally, let $\Sigma$ be a complete theory and define $K_{\Sigma}=\{\mathcal{A} \models \Sigma$ : the universe of $\mathcal{A}$ is $\omega$. Then isomorphisms between elements of $K_{\Sigma}$ are permutations of $\omega$, and the symmetric group $\operatorname{Sym}(\omega)$ acts on $K_{\Sigma}$ in the natural way. Similarly, elementary embeddings between elements of $K_{\Sigma}$ are injective functions of $\omega$. Vaught's conjecture can then be rephrased as follows: if the cardinality of orbits of $\operatorname{Sym}(\omega)$ on $K_{\Sigma}$ is uncountable, then this cardinality is the continuum. Problems 1.3 and 1.4 may be rephrased in a similar, but more complicated way. (The main difference is that in these cases, we cannot speak about orbits, since the injective functions of $\omega$ only form a monoid.) After making the role of $\operatorname{Sym}(\omega)$ explicit, it is natural to study what happens if we replace it with some simpler group or monoid.

For a monoid $S \subseteq{ }^{\omega} \omega$, we define $I(\Sigma, S)$ to be the supremum of the cardinalities of sets containing countable models of $\Sigma$ which cannot be elementarily embedded into each other by elements of $S$; more formally:

$$
I(\Sigma, S)=\sup \left\{|K|: K \subseteq K_{\Sigma} \text { and no } \varrho \in S\right.
$$

is an elementary embedding between any two elements of $K$ \}.

Endow $\omega$ with the discrete topology and ${ }^{\omega} \omega$ with the obtained product topology. Then a set $S \subseteq{ }^{\omega} \omega$ is defined to be $\sigma$-compact iff it is a union of a countable family of compact subsets of ${ }^{\omega} \omega$. The main results of this paper are as follows.

Theorems 5.8 and 5.9 In the case of languages both with and without equality, we have: if $S$ is a $\sigma$-compact monoid of injective functions of $\omega$, then

$$
I(\Sigma, S)>\aleph_{0} \text {, implies } I(\Sigma, S)=2^{\aleph_{0}} .
$$

Notice that if $S$ is the monoid of injective functions of $\omega$, then $I(\Sigma, S)=I^{\prime}\left(\Sigma, \aleph_{0}\right)$, and that $I(\Sigma, \operatorname{Sym}(\omega))=I\left(\Sigma, \aleph_{0}\right)$. Hence, by studying $I(\Sigma, S)$, one may obtain results for all the four problems formulated above.

Our approach is similar in spirit to some investigations in [2], but there is the following essential difference. In [2], the actions of Polish groups on the Borel subsets of certain spaces are investigated. Polish groups are complete metric spaces; we do not assume that the monoid $S$ occurring in our statements is a group, or that it is a complete space. Since we assume only that $S$ is a monoid (and not necessarily a group), classical results of descriptive set theory, (on actions of Polish groups, say,) are no longer available. In this respect, our approach is different from the traditional one. However, as we will see, the cylindric algebraic methods initiated in [17] may 
be adapted to this setting too. So our approach at the technical level is also different from the methods established in [2].

The rest of the paper is organized as follows. At the end of this section, we sum up our system of notation. Section 2 contains the necessary preliminaries from the theory of cylindric and quasi-polyadic algebras. This section has a survey character: all the results in this section are well known, and so, in order to keep this paper self contained and available to readers not so familiar with algebraic logic, instead of proofs, we provide explanations that are sometimes more detailed than usual. In Scection 3, we deal with representations of quasi-polyadic algebras. The cylindric algebraic analogues of the theorems in this sections are known; here we adapt them to the context of quasi-polyadic algebras. In Section 4, we characterize elementary embeddings in terms of algebraic logic. We consider the case without equality in the first subsection, and the case with equality in the second one. For completeness, we also examine in the second subsection the cylindric algebraic versions of results presented in Section 3. Some of them are already known, but based on our results in Section 3, they may be established quickly. In addition, Lemmas 4.2 and 4.3 are slightly different from the corresponding results of [17] (see Theorem 2.1 therein). In Section 5, (after further technical preparation,) we present the main results of the paper in Theorems 5.8 and 5.9. Finally, in Section 6, we suggest some further directions of possible investigation where the methods used in the present paper seem to be applicable.

\section{Notation}

Our system of notation is mostly standard, but the following list may be useful. Throughout, $\omega$ denotes the set of natural numbers, and for every $n \in \omega$ we have $n=\{0,1, \ldots, n-1\}$. Let $A$ and $B$ be sets. Then ${ }^{A} B$ denotes the set of functions whose domain is $A$ and whose range is a subset of $B$. In addition, $|A|$ denotes the cardinality of $A$. If $\kappa$ is a cardinal, then $[A]^{\kappa}$ denotes the set of subsets of $A$ which are of cardinality $\kappa$, and $\mathcal{P}(A)$ denotes the power set of $A$, that is, the set which consists of all the subsets of $A$. For any distinct elements $i, j$ from a given set $U$, $[i / j] \in{ }^{U} U$ is the function on $U$ which maps $i$ to $j$ and leaves every other element fixed. In addition, $I d_{U}$ denotes the identity function on $U$. Throughout, we use function composition in such a way that the rightmost factor acts first. That is, for functions $f, g$ we define $f \circ g(x)=f(g(x))$. If $f: A \longrightarrow B$ is a fuction and $X \subseteq A$, then $f^{*}(X)=\{f(x): x \in X\}$. Moreover, $f^{-1}: \mathcal{P}(B) \longrightarrow \mathcal{P}(A)$ acts between the powersets. 


\section{Preliminaries from the theories of cylindric and quasi-polyadic algebras}

This section surveys the basic properties of cylindric and quasi-polyadic algebras needed in later sections. We do not assume the reader is familiar with these classes of algebras, hence our presentation may be more detailed than usual. However, familiarity with cylindric and quasi-polyadic algebras may be an advantage of the reader in later sections. The definitions and propositions of this section, along with proofs, can be found in either [9] or [10].

Cylindric algebras emerged in the first half of the twentieth century, due to the work of Alfred Tarski and his students. Their original intention with this theory was to provide an algebraic treatment of first order logic (with equality), just as Boolean algebras do for sentential calculus. In fact, a cylindric algebra is a Boolean algebra equipped with additional operations $c_{i}$ corresponding to existential quantifiers and $d_{i j}$ corresponding to equality relations. More precisely, Let $\alpha$ be an ordinal. Then an $\alpha$-dimensional cylindric algebra is an algebraic structure of the form

$$
\mathcal{A}=\left\langle A, \wedge,-, c_{i}, d_{i j}\right\rangle_{i, j \in \alpha}
$$

where the $c_{i}$ are unary operations and the $d_{i j}$ are constants and $\mathcal{A}$ satisfies certain equational posulates (see e.g. Definition 1.1.1 of [9]). We do not recall these postulates, because we do not use their concrete forms below. CA and $\mathrm{CA}_{\alpha}$ denote the class of all cylindric algebras and the class of cylindric algebras of dimension $\alpha$, respectively. There are two main methods of constructing cylindric algebras, called "algebraizing syntax" and "algebraizing semantics".

We start by describing algebraizing syntax. Suppose $L$ is a first order language with equality and let $\Sigma$ be a theory in $L$. Two formulas $\varphi$ and $\psi$ are defined to be equivalent $\bmod \Sigma$, in symbols $\varphi \equiv_{\Sigma} \psi$, iff $\Sigma \models \forall(\varphi \Leftrightarrow \psi)$, where $\forall(\varphi \Leftrightarrow \psi)$ is the universal closure of $\varphi \Leftrightarrow \psi$. Clearly, $\equiv_{\Sigma}$ is an equivalence relation on the set of formulas Form $_{L}$. Quotienting the formula algebra (the completely free algebra) with this equivalence relation, we obtain an $\omega$-dimensional cylindric algebra which is denoted by $\mathrm{CA}(\Sigma)$ and sometimes called the Lindenbaum algebra of $\Sigma$.

Algebraizing semantics may be described as follows. Keeping the notation of the previous paragraph, let $\mathcal{M}$ be a model of $\Sigma$ and for a formula $\varphi$ let us denote by $\|\varphi\|^{\mathcal{M}}$ (or simply, by $\|\varphi\|$ ) the "relation" defined by $\varphi$ in $\mathcal{M}$, ie. $\|\varphi\|^{\mathcal{M}}=\left\{f \in{ }^{\omega} M: \mathcal{M} \models \varphi[f]\right\}$. Let

$$
A=\left\{\|\varphi\|: \varphi \in \operatorname{Form}_{L}\right\}
$$

Then $A$ is closed under set theoretic intersection and complementation. Moreover, $A$ may be expanded to an $\omega$-dimensional cylindric algebra by adding the following 
operations $c_{i}, d_{i j}(i, j \in \omega)$ to its similarity type (under which $A$ is also closed):

$$
c_{i}\|\varphi\|=\left\|\exists v_{i} \varphi\right\| \quad \text { and } \quad d_{i j}=\left\|v_{i}=v_{j}\right\| .
$$

The algebra obtained in this way is denoted by $\operatorname{Cs}(\mathcal{M})$ and is called the cylindric set algebra of $\mathcal{M}$.

Suppose $\varphi, \psi \in \operatorname{Form}_{L}$ and $\psi$ can be obtained from $\varphi$ by substituting the variable $v_{j}$ for $v_{i}$ (where both $v_{i}$ and $v_{j}$ are assumed to be unbound variables in $\varphi$ ). Let $s_{j}^{i} x$ be the cylindric term $c_{i}\left(x \wedge d_{i j}\right)$. Then in $\operatorname{CA}(\Sigma)$ we have $\psi / \equiv_{\Sigma}=s_{j}^{i}\left(\varphi / \equiv_{\Sigma}\right)$ and in $\operatorname{Cs}(\mathcal{M})$ we have $\|\psi\|=s_{j}^{i}\|\varphi\|$. For $i, j \in \omega$, the cylindric algebraic terms $s_{j}^{i}$ are called substitutions.

When one wants to apply the same process to theories in languages do not containing the equality symbol, substitution operations are no longer term definable. To overcome this difficulty, the $s_{j}^{i}$ are defined to be basic operations when algebraizing equality free logics. More concretely, an $\alpha$-dimensional quasi-polyadic algebra is an algebraic structure of the form

$$
\mathcal{A}=\left\langle A, \wedge,-, c_{i}, s_{j}^{i}\right\rangle_{i, j \in \alpha}
$$

satisfying certain equational postulates. Here, the $c_{i}$ correspond to existential quantifiers, and the $s_{j}^{i}$ correspond to substitution operations. The class of $\alpha$ dimensional quasi-polyadic algebras is denoted by $\mathrm{QPA}_{\alpha}$. Algebraizing syntax and semantics may be defined similarly to the cylindric case; the resulting algebras are denoted by $\mathrm{QPA}(\Sigma)$ and by $\mathrm{Qs}(\mathcal{M})$ respectively.

Let $\mathcal{A}$ be an $\alpha$-dimensional cylindric or quasi-polyadic algebra and let $x \in A$. The dimension set $\Delta x$ of $x$ is defined to be $\Delta x=\left\{i \in \alpha: x \neq c_{i} x\right\}$. Intuitively, $\Delta x$ is the set of variables $x$ (as a "formula") really depends on. $\mathcal{A}$ is defined to be locally finite dimensional iff every element of $\mathcal{A}$ has a finite dimension set. Algebras obtained by the algebraization processes described above are locally finite dimensional, because each formula contains only a finite number of variables. The class of locally finite dimensional algebras is denoted by Lf in the cylindric case, and LfQPA in the quasi-polyadic case.

Simultaneous substitutions can also be generalized as follows. Let $\tau \in{ }^{\omega} \omega$ and for every $i \in \omega$ let's substitute $v_{\tau(i)}$ for $v_{i}$ simultaneously. As explained in Definitions 1.11.9 and 1.11.13 of [9], these kind of generalized substitutions can be introduced in every $L f$, the derived function is denoted by $s_{\tau}^{+}$.

Let $U$ be a set. Then the full cylindric set algebra on $U$ of dimension $\alpha$ is the structure $\left\langle\mathcal{P}\left({ }^{\alpha} U\right), \cap,-, C_{i}, D_{i, j}\right\rangle_{i, j \in \alpha}$, where $\cap$ is set theoretical intersection, - is complementation (w.r.t. ${ }^{\alpha} U$ ), and for any $X \subseteq{ }^{\alpha} U$ and $i, j \in \alpha$,

$$
C_{i} X=\left\{s \in{ }^{\alpha} U:(\exists z \in X)\left(s_{\mid \alpha-\{i\}}=z_{\mid \alpha-\{i\}}\right)\right\} \text { and }
$$




$$
D_{i, j}=\left\{s \in{ }^{\alpha} U: s(i)=s(j)\right\} .
$$

The class $\mathrm{Cs}_{\alpha}$ of $\alpha$ dimensional cylindric set algebras is defined to be the class of all subalgebras of $\alpha$ dimensional full cylindric set algebras. Similarly, the full quasipolyadic set algebra on $U$ of dimension $\alpha$ is the structure $\left\langle\mathcal{P}\left({ }^{\alpha} U\right), \cap,-, C_{i}, S_{j}^{i}\right\rangle_{i, j \in \alpha}$, where $\cap,-$ and $C_{i}$ are as before, and for any $X \subseteq{ }^{\alpha} U$ and $i, j \in \alpha$,

$$
S_{j}^{i} X=\left\{s \in{ }^{\alpha} U: s \circ[i / j] \in X\right\} .
$$

The class $\mathrm{Qs}_{\alpha}$ of $\alpha$ dimensional quasi-polyadic set algebras is defined to be the class of all subalgebras of $\alpha$ dimensional full quasi-polyadic set algebras. Note that Cs $\subseteq$ CA and Qs $\subseteq$ QPA, that is, cylindric set algebras are cylindric algebras, and quasi-polyadic set algebras are quasi-polyadic algebras (of the appropriate dimension). Also, if $M$ is a model for a language with equality, then $\operatorname{Cs}(\mathcal{M})$ is a cylindric set algebra, and in the equality-free case, $\operatorname{Qs}(\mathcal{M})$ is a quasi-polyadic set algebra.

An element $X$ of an $\alpha$ dimensional cylindric or quasi-polyadic set algebra $\mathcal{A}$ is defined to be regular iff for every $s, z \in{ }^{\alpha} U$ we have

$$
s \in X \text { and } s_{\mid \Delta(X)}=z_{\mid \Delta(X)} \text { imply } z \in X .
$$

A cylindric or quasi-polyadic set algebra is regular iff all of its elements are regular. The class of $\alpha$ dimensional regular cylindric or quasi-polyadic set algebras is denoted by $\mathrm{Cs}_{\alpha}^{R e g}$ and $\mathrm{Qs}_{\alpha}^{R e g}$, respectively. If $\mathcal{M}$ is a model for $L$, then $\operatorname{Cs}(\mathcal{M})$ and $\operatorname{Qs}(\mathcal{M})$ are regular, because if $\varphi$ is a formula of $L$ and $s \in{ }^{\omega} U$ is a valuation (where $U$ is the universe of $\mathcal{M}$ ), then whether $\mathcal{M} \models \varphi[s]$ holds or not depends only on the value of $s$ at those $k$ for which $\mathcal{M} \not \forall \forall\left(\exists v_{k} \varphi \Leftrightarrow \varphi\right)$, (where $\forall\left(\exists v_{k} \varphi \Leftrightarrow \varphi\right)$ is the universal closure of $\left.\exists v_{k} \varphi \Leftrightarrow \varphi\right)$. Equivalently, $s \in\|\varphi\|^{\mathcal{M}}$ depends only on $s_{\mid \Delta\left(\|\varphi\|^{\mathcal{M}}\right)}$.

We conclude this section by recalling some well known properties of dimension sets, cylindrifications and substitutions (without proofs).

Proposition 2.1 Suppose $\mathcal{A} \in \mathrm{CA}_{\alpha}$ or $\mathcal{A} \in \mathrm{QPA}_{\alpha}$. For all $x, y \in A$ and $i<\alpha$, we have

1. $\Delta 0=\Delta 1=\emptyset$

2. $\Delta(-x)=\Delta x$,

3. $\Delta(x \vee y) \subseteq \Delta x \cup \Delta y$,

4. $\Delta(x \wedge y) \subseteq \Delta(x) \cup \Delta y$,

5. $\Delta\left(c_{i} x\right) \subseteq \Delta x-\{i\}$.

Proposition 2.2 Suppose $\mathcal{A} \in \mathrm{Lf}_{\alpha}$ or $\mathcal{A} \in \mathrm{LfQPA}_{\alpha}$. For any $x \in A, i, j \in \alpha$ and $\tau, \sigma \in{ }^{\alpha} \alpha$ we have:

1. if $\{n \in \alpha: n \neq \tau(n)\}$ is finite, then $s_{\tau}^{+}$is a term function.

2. $s_{[i / j]}^{+}=s_{j}^{i}$, 
3. $s_{I d_{\alpha}}^{+}=I d_{A}$,

4. $s_{\tau}^{+}$is a Boolean endomorphism,

5. $s_{\sigma \circ \tau}^{+} x=s_{\sigma}^{+} s_{\tau}^{+} x$,

6. if $\sigma_{\mid \alpha-\{i\}}=\tau_{\mid \alpha-\{i\}}$, then $s_{\sigma}^{+} c_{i} x=s_{\tau}^{+} c_{i} x$,

7. if $\sigma_{\mid \Delta x}=\tau_{\mid \Delta x}$, then $s_{\sigma}^{+} x=s_{\tau}^{+} x$,

8. if $\tau^{-1}\{j\}=\{i\}$, then $c_{j} s_{\tau}^{+} x=s_{\tau}^{+} c_{i} x$.

9. $\Delta\left(s_{\tau}^{+} x\right) \subseteq \tau^{*} \Delta x$.

Proposition 2.3 Let $\mathcal{A} \in \mathrm{Lf}_{\alpha}$, and $\tau \in{ }^{\alpha} \alpha$. Then $s_{\tau}^{+} d_{i j}=d_{\tau(i) \tau(j)}$.

Suppose $\Sigma$ is a theory in a language $L$ with equality. Then $h: \mathrm{CA}(\Sigma) \longrightarrow$ $\operatorname{Cs}(\mathcal{M}), \varphi / \equiv_{\Sigma} \mapsto\|\varphi\|$ is a surjective homomorphism. Thus, $\operatorname{Cs}(\mathcal{M})$ is a homomorphic image of $\operatorname{CA}(\Sigma)$, and, as we have already mentioned above, $\operatorname{Cs}(\mathcal{M})$ is a locally finite dimensional and regular cylindric set algebra. Conversely, an element $\mathcal{A} \in$ $\mathrm{Lf}_{\omega} \cap \mathrm{Cs}_{\omega}^{\text {Reg }}$, together with a distinguished set of its generators, determines a model for some language (this fact is well known, but a proof is recalled in Section 2 of [17]). This model satisfies $\Sigma$ (and is a model for $L$ ) iff there is a homomorphism from $\operatorname{CA}(\Sigma)$ onto $\mathcal{A}$ mapping the distinguished set of generators of $\mathrm{CA}(\Sigma)$ (ie. $\left\{R / \equiv_{\Sigma}: R \in L\right\}$ ) onto the distinguished set of generators of $\mathcal{A}$.

Similarly, if $\Sigma$ is a theory in a language without equality, there is also the above one-one corraspondance between models of $\Sigma$ and surjective homomorphisms from QPA $(\Sigma)$ onto elements of $\mathrm{LfQPA}_{\omega} \cap \mathrm{Qs}_{\omega}^{\text {Reg }}$. Consequently, the problem of finding all the possible countable models of $\Sigma$ is equivalent to finding all homomorphisms from either $\mathrm{CA}(\Sigma)$ or QPA $(\Sigma)$ onto some locally finite dimensional and regular set algebra with a countable base.

\section{Representations of certain quasi-polyadic alge- bras}

The representation theory of cylindric and quasi-polyadic algebras has a great tradition, for more recent investigations we refer to e.g. [7],[8],[14],[15],[16] and [20].

In Remark 3.2.9 of [10] and in [1] (see also [17]), a method of constructing homomorphisms from an $\mathrm{Lf}_{\omega}$ with a countable universe onto some $\mathrm{Lf}_{\omega} \cap \mathrm{Cs}_{\omega}^{\text {Reg }}$ is given. Below, we adapt this method for quasi-polyadic algebras. To do so, we need some further preperation.

Given a Boolean algebra $\mathcal{B}$, let $\mathcal{U}(\mathcal{B})$ denote the set of ultrafilters on $\mathcal{B}$. Recall that the Stone topology $\mathcal{T}$ on $\mathcal{U}(\mathcal{B})$ is defined as follows: for each $x \in B$, let $N_{x}$ be defined as $N_{x}=\{\mathcal{F} \in \mathcal{U}(\mathcal{B}): x \in \mathcal{F}\}$; then $\mathcal{T}$ is the topology generated by the basis $\left\{N_{x}: x \in B\right\}$. This space is denoted by $\mathcal{B}^{*}$ and sometimes called the dual of $\mathcal{B}$. Note that the Stone space $\mathcal{B}^{*}=\langle\mathcal{U}(\mathcal{B}), \mathcal{T}\rangle$ is a compact Hausdorff space, and the basic sets $N_{x}$ are clopen. Suppose that for some element $x$ and subset $Y$ of $B$ we 
have $x=\sup Y$. We will say an ultrafilter $\mathcal{F} \in \mathcal{U}(\mathcal{B})$ preserves $Y$ iff $x \in \mathcal{F}$ implies $y \in \mathcal{F}$ for some $y \in Y$.

Let $\mathcal{A}$ be an LfQPA $_{\omega}$ that has a countable universe. Note that if $\Sigma$ is a theory in a language without equality, then $\mathrm{QPA}(\Sigma)$ satisfies these requirements. Following [17], we denote by $\mathcal{U}_{i, x}(\mathcal{A})$ the set of ultrafilters on $\mathcal{A}$ that preserve $\left\{s_{j}^{i} x: j<\omega\right\}$. We note that $c_{i} x=\sup \left\{s_{j}^{i} x: j \in \omega\right\}$ by Theorem 1.11.6 of [9], and so,

$$
\begin{aligned}
\mathcal{U}_{i, x}(\mathcal{A}) & =\left\{\mathcal{F} \in \mathcal{U}(\mathcal{A}): c_{i} x \in \mathcal{F} \text { implies } s_{j}^{i} x \in \mathcal{F} \text { for some } j<\omega\right\} \\
& =N_{-c_{i} x} \cup \bigcup_{j<\omega} N_{s_{j}^{i} x} .
\end{aligned}
$$

Let $\mathcal{H}(\mathcal{A})$ denote the set of those ultrafilters of $\mathcal{A}$ that preserve $\left\{s_{j}^{i} x: j<\omega\right\}$ for all $x \in A$ and $i<\omega$, that is

$$
\mathcal{H}(\mathcal{A})=\bigcap_{i<\omega, x \in A} \mathcal{U}_{i, x}(\mathcal{A})
$$

For completeness, we note that $\mathcal{H}(\mathcal{A})$ is nonempty. This follows from the Baire category theorem and the fact that for each $i$ and $x$, the complement of $\mathcal{U}_{i, x}(\mathcal{A})$ is a nowhere dense subset of $\mathcal{A}^{*}$. We do not prove this, because our results below imply immediately that $\mathcal{H}(\mathcal{A})$ is nonempty.

Take any $\mathcal{F} \in \mathcal{H}(\mathcal{A})$, and for any $x \in A$ define the function $\operatorname{rep}_{\mathcal{F}}$ to be

$$
\operatorname{rep}_{\mathcal{F}}(x)=\left\{\tau \in{ }^{\omega} \omega: s_{\tau}^{+} x \in \mathcal{F}\right\} \text {. }
$$

In the next two lemmas, we show that $\operatorname{rep}_{\mathcal{F}}$ is a homomorphism from $\mathcal{A}$ onto some locally finite dimensional and regular $\mathrm{Qs}_{\omega}$, and that the converse is also true: any such homomorphism can be obtained as a result of this construction. Lemma 3.1 is the quasi-polyadic analogue of some results presented in Remark 3.2.9 of [10] and in [1], while Lemma 3.2 is the quasi-polyadic analogue of Lemma 2.1 of [17] (where it was presented for cylindric algebras in a more complicated form).

Lemma 3.1 For an arbitrary $\mathcal{F} \in \mathcal{H}(\mathcal{A}), \operatorname{rep}_{\mathcal{F}}$ is a homomorphism onto some $\mathrm{LfQPA}_{\omega} \cap \mathrm{Qs}_{\omega}^{\text {Reg }}$ with base $\omega$. Furthermore, for all $\mathcal{F}_{0}, \mathcal{F}_{1} \in \mathcal{H}(\mathcal{A})$ we have $\operatorname{rep}_{\mathcal{F}_{0}}=$ $\operatorname{rep}_{\mathcal{F}_{1}}$ iff $\mathcal{F}_{0}=\mathcal{F}_{1}$.

Proof. It is easy to see, using Proposition 2.2 that

(i) $\operatorname{rep}_{\mathcal{F}}$ preserves $-\wedge$ and $s_{j}^{i}$ for all $i, j \in \omega$, and

(ii) $\operatorname{rep}_{\mathcal{F}}(x)$ is regular for each $x \in A$; (this is because if $\tau \in \operatorname{rep}_{\mathcal{F}}(x)$ and $\tau_{\mid \Delta x}=\sigma_{\mid \Delta x}$, then, by 7 . of Proposition 2.2 we have $s_{\sigma}^{+} x=s_{\tau}^{+} x \in \mathcal{F}$, whence $\left.\sigma \in \operatorname{rep}_{\mathcal{F}}(x)\right)$.

To show that $C_{i} \operatorname{rep}_{\mathcal{F}}(x)=\operatorname{rep}_{\mathcal{F}}\left(c_{i} x\right)$, first suppose that $\tau \in C_{i} \operatorname{rep}_{\mathcal{F}}(x)$. This means that for some $\sigma \in{ }^{\omega} \omega$, we have $\sigma_{\mid \omega-\{i\}}=\tau_{\mid \omega-\{i\}}$ and $s_{\sigma}^{+} x \in \mathcal{F}$. Combining this with 
4. and 6. of Proposition 2.2, we obtain $\mathcal{F} \ni s_{\sigma}^{+} c_{i} x=s_{\tau}^{+} c_{i} x$. Hence $\tau \in \operatorname{rep}_{\mathcal{F}}\left(c_{i} x\right)$. Now, suppose $\tau \in \operatorname{rep}_{\mathcal{F}}\left(c_{i} x\right)$. First, take a $\rho \in{ }^{\omega} \omega$ such that

$$
\rho(j)= \begin{cases}\tau(j) & \text { if } j \in \Delta x-\{i\} \\ 0 & \text { if } j \in \omega-(\Delta x \cup\{i\}) \\ l & \text { for } j=i,\end{cases}
$$

where $l \in \omega-\left(\tau^{*} \Delta x \cup\{0\}\right)$. We have $\rho_{\mid \Delta x-\{i\}}=\tau_{\mid \Delta x-\{i\}}$. Also, $\tau^{-1}\{l\}=\{i\}$, and so 5. of Proposition 2.1 and $\%$. and 8. of Proposition 2.2 give us

$$
s_{\tau}^{+} c_{i} x=s_{\rho}^{+} c_{i} x=c_{l} s_{\rho}^{+} x .
$$

Since $c_{l} s_{\rho}^{+} x=s_{\tau}^{+} c_{i} x \in \mathcal{F}$ and $\mathcal{F} \in \mathcal{H}(\mathcal{A})$, there is a $k \in \omega$ such that $s_{k}^{l} s_{\rho}^{+} x \in \mathcal{F}$, that is $[l / k] \circ \rho \in \operatorname{rep}_{\mathcal{F}}(x)$.

Let $\sigma=([l / k] \circ \rho)_{\mid \Delta x} \cup I d_{\omega-\Delta x}$. Notice that $\Delta \operatorname{rep}_{\mathcal{F}}(x) \subseteq \Delta x$. (This is because $\operatorname{rep}_{\mathcal{F}}(x) \subseteq C_{i} \operatorname{rep}_{\mathcal{F}}(x) \subseteq \operatorname{rep}_{\mathcal{F}}\left(c_{i} x\right)$ for all $i \in \omega$. So, if $i \notin \Delta(x)$, then it follows, that $\operatorname{rep}_{\mathcal{F}}(x)=C_{i} r e p_{\mathcal{F}}(x)$.) Therefore, since $\operatorname{rep}_{\mathcal{F}}(x)$ is regular, $\sigma \in \operatorname{rep}_{\mathcal{F}}(x)$. Hence, we have

$$
C_{i} \operatorname{rep}_{\mathcal{F}}(x) \ni \sigma_{\mid \omega \smile\{i\}} \cup I d_{\{i\}}=\tau_{\mid \Delta x \smile\{i\}} \cup I d_{(\omega-\Delta x) \cup\{i\}} .
$$

Since $\operatorname{rep}_{\mathcal{F}}(x)$ is regular, so is $C_{i} \mathrm{rep}_{\mathcal{F}}(x)$, and so $\tau \in C_{i} \operatorname{rep}_{\mathcal{F}}(x)$.

Consequently, $\operatorname{rep}_{\mathcal{F}}$ is a homomorphism from $\mathcal{A}$ onto some quasi-polyadic set algebra $\mathcal{B}$ with base $\omega . \mathcal{B}$ is locally finite dimensional because $\mathcal{A}$ is, and is regular, because each of its elements are. The second statement is easily seen.

Next, we show that every homomorphism from $\mathcal{A}$ onto some locally finite dimensional $\mathrm{Qs}_{\omega}^{\text {Reg }}$ with base $\omega$ can be obtained as a result of the previous construction.

Lemma 3.2 Let $\mathcal{B} \in \mathrm{LfQPA}_{\omega} \cap \mathrm{Qs}_{\omega}^{\text {Reg }}$ with base $\omega$. For any homomorphism $h$ from $\mathcal{A}$ onto $\mathcal{B}$, there is an $\mathcal{F} \in \mathcal{H}(\mathcal{A})$ such that $h=\operatorname{rep}_{\mathcal{F}}$.

Proof. Consider the following subset of $A$ :

$$
\mathcal{F}=\left\{x \in A: I d_{\omega} \in h(x)\right\} .
$$

It is not hard to check that $\mathcal{F}$ is an ultrafilter. We will show that $\mathcal{F} \in \mathcal{H}(\mathcal{A})$, and that $\operatorname{rep}_{\mathcal{F}}=h$.

Let $x \in A$ and $i \in \omega$. Suppose we have $c_{i} x \in \mathcal{F}$. This means that $I d_{\omega} \in h\left(c_{i} x\right)=$ $C_{i} h(x)$. Therefore $[i / k] \in h(x)$ for some $k \in \omega$, implying that

$$
I d_{\omega} \in\{\sigma: \sigma \circ[i / k] \in h(x)\}=S_{k}^{i} h(x)=h\left(s_{k}^{i} x\right)
$$

and so $s_{k}^{i} x \in \mathcal{F}$. Consequently, $\mathcal{F} \in \mathcal{U}_{i, x}(\mathcal{A})$. Because the above $x$ and $i$ were chosen arbitrarily, $\mathcal{F} \in \mathcal{H}(\mathcal{A})$.

Finally, observe that for any $x \in A$ and $\tau \in{ }^{\omega} \omega$, we have

$$
\begin{aligned}
\tau \in \operatorname{rep}_{\mathcal{F}}(x) \text { iff } s_{\tau}^{+} x \in \mathcal{F} & \text { iff } I d_{\omega} \in h\left(s_{\tau}^{+} x\right)=S_{\tau}^{+} h(x) \\
& \text { iff } I d_{\omega} \circ \tau \in h(x) \text { iff } \tau \in h(x) .
\end{aligned}
$$


This means that $h=\operatorname{rep}_{\mathcal{F}}$, as desired.

\section{Elementary embeddings and representations over a countable base set}

In this paper, we are interested in the countable models of a consistent theory $\Sigma$ only up to elementary embeddability; that is, we do not want to distinguish between two models $\mathcal{M}$ and $\mathcal{N}$ - or their corresponding ultrafilters - if either there is an elementary embedding $\rho$ of $\mathcal{M}$ into $\mathcal{N}$ or vice versa. Our goal is to characterize the ultrafilters that lead to "the same" models; this will be done in Theorem 4.1 in the equality-free case and in Theorem 4.5 in the case with equality.

\subsection{The case without equality}

Throughout this subsection, we will suppose $\mathcal{A}=\mathrm{QPA}(\Sigma)$ for some consistent theory $\Sigma$ in a language $L$ without equality. Recall from Section 1 that

$$
K_{\Sigma}=\{\mathcal{M} \models \Sigma: \text { the universe of } \mathcal{M} \text { is } \omega\} .
$$

Due to Lemmas 3.1 and 3.2 and the paragraph right after Proposition 2.3, there is a one-one correspondence between the models in $K_{\Sigma}$ and the ultrafilters in $\mathcal{F} \in$ $\mathcal{H}(\mathcal{A})$. More specifically, if $\mathcal{M} \in K_{\Sigma}$, then there is an $\mathcal{F} \in \mathcal{H}(\mathcal{A})$ such that $\operatorname{rep}_{\mathcal{F}}$ is a homomorphism from $\mathcal{A}$ onto $\mathrm{Qs}(\mathcal{M})$. Conversely, if $\mathcal{F} \in \mathcal{H}(\mathcal{A})$, then $\operatorname{rep}_{\mathcal{F}}$ is a homomorphism from $\mathcal{A}$ onto some $\mathcal{B} \in \mathrm{LfQPA}_{\omega} \cap \mathrm{Qs}_{\omega}^{\text {Reg }}$ with base $\omega$, and so $\mathcal{B}=\operatorname{Qs}(\mathcal{M})$ for some model $\mathcal{M}$ of $\Sigma$ with universe $\omega$. Note that in both cases, we have $\operatorname{rep}_{\mathcal{F}}\left(\varphi / \equiv_{\Sigma}\right)=\|\varphi\|^{\mathcal{M}}$. Consequently, instead of the models of $\Sigma$ with base $\omega$, we can work with $\mathcal{H}(\mathcal{A})$. Now, we will determine which of its elements lead to pairwise non-elementarily embeddable models.

Suppose $\rho$ is a bijection between the sets $U$ and $W$. For every $X \subseteq{ }^{\alpha} U$ let

$$
f(X)=\{\rho \circ \sigma: \sigma \in X\}
$$

If $\mathcal{B} \in \mathrm{Qs}_{\alpha}$ with base $U$, then $f_{\mid B}$ is clearly an isomorphim onto some $\mathrm{Qs}_{\alpha}$. Isomorphisms of this form are called base isomorphisms.

Suppose $\mathcal{B}$ and $\mathcal{C}$ are $\alpha$ dimensional quasi-polyadic set algebras with bases $U$ and $V$, and $U \supseteq V$. Suppose $\Phi$ is an isomorphism between them. If for every $X \in B$ we have

$$
\Phi(X)=X \cap{ }^{\alpha} V
$$

then $\Phi$ is called an ext.-isomorphism. We call a function $h=f \circ \Phi$ an ext.-baseisomorphism iff $f$ is a base isomorphism, and $\Phi$ is an ext.-isomorphism. (We note 
that a slightly different, although equivalent definition of ext.-base-isomorphisms is given in 3.1.41 of [10].) Base isomorphisms and ext.-isomorphisms between cylindric set algebras are defined similarly to the quasi-polyadic case.

In the case of cylindric algebras (i.e. the case of languages with equality), the characterization of isomorphism and elementary embeddability with the previous concepts is well known: suppose $\mathcal{M}$ and $\mathcal{N}$ are models for a language $L$ that contains the equality symbol. Then

- $\mathcal{M} \cong \mathcal{N}$ iff $\operatorname{Cs}(\mathcal{M})$ is base isomorphic to $\operatorname{Cs}(\mathcal{N})$ with the natural generators preserved, ie. the image of $\|R\|^{\mathcal{M}}$ is $\|R\|^{\mathcal{N}}$ for all $R \in L$.

- $\mathcal{M} \succcurlyeq \mathcal{N}$ iff $\operatorname{Cs}(\mathcal{M})$ is ext.-isomorphic to $\operatorname{Cs}(\mathcal{N})$ with the natural generators preserved, (see 10. of Remark 4.3.86 of [10]). Thus $\mathcal{N}$ is elementarily embeddable into $\mathcal{M}$ iff $\operatorname{Cs}(\mathcal{M})$ and $\operatorname{Cs}(\mathcal{N})$ are ext.-base-isomorphic with the natural generators preserved.

In Definition 3.1 and Theorem 3.2 of [17], a characterization of ultrafilters that correspond to isomorphic models is given (although only in the case with equality). The next theorem is motivated by this characterization, and the above. ( $\mathcal{A}$ is still $\operatorname{QPA}(\Sigma)$, where $\Sigma$ is a theory in an equality-free language $L$.)

Theorem 4.1 Let $\mathcal{M}_{0}, \mathcal{M}_{1} \in K_{\Sigma}$. Suppose $\mathcal{F}_{0}, \mathcal{F}_{1} \in \mathcal{H}(\mathcal{A})$ are such that rep $\operatorname{F}_{\mathcal{F}_{i}}$ is a homomorphism from $\mathcal{A}$ onto $\mathrm{Qs}_{\omega}\left(\mathcal{M}_{i}\right)(i=0,1)$. Suppose $\rho: \omega \longrightarrow \omega$ is injective, and let $U=$ Range $\rho$. For each $X \subseteq{ }^{\omega} \omega$ let $f_{\rho}(X)=\{\rho \circ \sigma: \sigma \in X\}$, and for each $X \in \mathrm{Qs}_{\omega}\left(\mathcal{M}_{1}\right)$ let $\Phi(X)=X \cap{ }^{\omega} U$. Then, the following are equivalent:

1. $\rho: \mathcal{M}_{0} \preccurlyeq \mathcal{M}_{1}$,

2. $f_{\rho} \circ \operatorname{rep}_{\mathcal{F}_{0}}=\Phi \circ \operatorname{rep}_{\mathcal{F}_{1}}$,

3. For each $x \in A$ we have $x \in \mathcal{F}_{0}$ iff $s_{\rho}^{+} x \in \mathcal{F}_{1}$, (that is, $\left.\mathcal{F}_{0}=\left(s_{\rho}^{+}\right)^{-1} \mathcal{F}_{1}\right)$.

Proof. To see that 1 . and 2. are equivalent, we will first prove the following claim.

Claim 4.1.1 $\rho: \mathcal{M}_{0} \preccurlyeq \mathcal{M}_{1}$ iff for every formula $\varphi$ of $L$ we have $f_{\rho}\left(\|\varphi\|^{\mathcal{M}_{0}}\right)=$ $\Phi\left(\|\varphi\|^{\mathcal{M}_{1}}\right)$.

\section{Proof of Claim 4.1.1}

$$
\begin{array}{ll}
\rho: \mathcal{M}_{0} \preccurlyeq \mathcal{M}_{1} & \text { iff } \\
\mathcal{M}_{0} \models \varphi[\sigma] \text { iff } \mathcal{M}_{1} \models \varphi[\rho \circ \sigma] \text { for every } \sigma \in{ }^{\omega} \omega \text { and } \varphi \in \operatorname{Form}_{L} & \text { iff } \\
\sigma \in\|\varphi\|^{\mathcal{M}_{0}} \text { iff } \rho \circ \sigma \in\|\varphi\|^{\mathcal{M}_{1}} \text { for every } \sigma \in{ }^{\omega} \omega \text { and } \varphi \in \operatorname{Form}_{L} & \text { iff } \\
\sigma \in\|\varphi\|^{\mathcal{M}_{0}} \text { iff } \rho \circ \sigma \in\|\varphi\|^{\mathcal{M}_{1}} \cap{ }^{\omega} U \text { for every } \sigma \in{ }^{\omega} \omega \text { and } \varphi \in \operatorname{Form}_{L} & \text { iff } \\
f_{\rho}\left(\|\varphi\|^{\mathcal{M}_{0}}\right)=\Phi\left(\|\varphi\|^{\mathcal{M}_{1}}\right) \text { for every } \varphi \in \text { Form }_{L} . &
\end{array}
$$


This completes the proof of Claim 4.1.1.

Because we have $\|\varphi\|^{\mathcal{M}_{i}}=\operatorname{rep}_{\mathcal{F}_{i}}\left(\varphi / \equiv_{\Sigma}\right)($ for $i=0,1)$ for every formula $\varphi$, and because the universe of $\mathcal{A}$ is $A=\left\{\varphi / \equiv_{\Sigma}: \varphi \in \operatorname{Form}_{L}\right\}$ we obtain, by Claim 4.1.1,

$$
\begin{array}{lc}
\rho: \mathcal{M}_{0} \preccurlyeq \mathcal{M}_{1} & \text { iff } \\
f_{\rho}\left(\|\varphi\|^{\mathcal{M}_{0}}\right)=\Phi\left(\|\varphi\|^{\mathcal{M}_{1}}\right) \text { for every } \varphi \in \operatorname{Form}_{L} & \text { iff } \\
f_{\rho}\left(\operatorname{rep}_{\mathcal{F}_{0}}\left(\varphi / \equiv_{\Sigma}\right)\right)=\Phi\left(\operatorname{rep}_{\mathcal{F}_{1}}\left(\varphi / \equiv_{\Sigma}\right)\right) \text { for every } \varphi \in \operatorname{Form}_{L} & \text { iff } \\
f_{\rho}\left(\operatorname{rep}_{\mathcal{F}_{0}}(x)\right)=\Phi\left(\operatorname{rep}_{\mathcal{F}_{1}}(x)\right) \text { for every } x \in A & \text { iff } \\
f_{\rho} \circ \operatorname{rep}_{\mathcal{F}_{0}}=\Phi \circ \operatorname{rep}_{\mathcal{F}_{1}} . &
\end{array}
$$

Now suppose the second statement holds, that is $f_{\rho} \circ \operatorname{rep}_{\mathcal{F}_{0}}(x)=\Phi \circ \operatorname{rep}_{\mathcal{F}_{1}}(x)$ for every $x \in A$. Then, for all $x \in A$ we have,

$$
\begin{array}{ll}
x \in \mathcal{F}_{0} & \text { iff } \\
s_{I d_{\omega}}^{+} x \in \mathcal{F}_{0} & \text { iff } \\
I d_{\omega} \in \operatorname{rep}_{\mathcal{F}_{0}}(x) & \text { iff } \\
\rho \circ I d_{\omega} \in f_{\rho} \circ \operatorname{rep}_{\mathcal{F}_{0}}(x) & \text { iff } \\
\rho \circ I d_{\omega} \in \Phi \circ \operatorname{rep}_{\mathcal{F}_{1}}(x)=\operatorname{rep}_{\mathcal{F}_{1}}(x) \cap{ }^{\omega} U & \text { iff } \\
\rho \in \operatorname{rep}_{\mathcal{F}_{1}}(x) & \text { iff } \\
s_{\rho}^{+} x \in \mathcal{F}_{1} . &
\end{array}
$$

Therefore the second statement implies the third. To see the other direction, suppose $x \in \mathcal{F}_{0}$ iff $s_{\rho}^{+} x \in \mathcal{F}_{1}$ for all $x \in A$. Then, for all $\tau \in{ }^{\omega} \omega$ we have

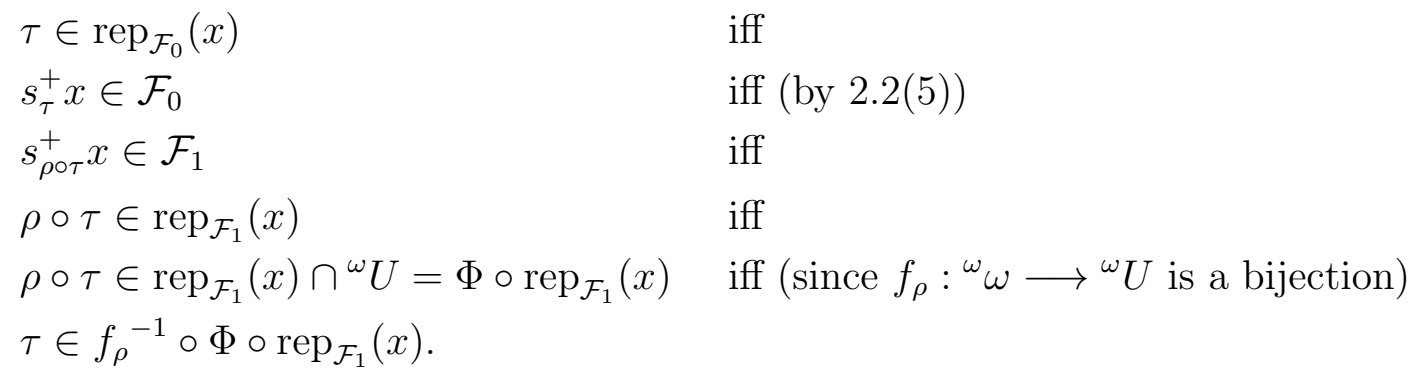

Thus we have $\operatorname{rep}_{\mathcal{F}_{0}}=f_{\rho}{ }^{-1} \circ \Phi \circ \operatorname{rep}_{\mathcal{F}_{1}}$, or equivalently, $f_{\rho} \circ \operatorname{rep}_{\mathcal{F}_{0}}=\Phi \circ \operatorname{rep}_{\mathcal{F}_{1}}$.

Note that Claim 4.1.1 implies that, (using the notation of the previous theorem) $\rho: \mathcal{M}_{0} \preccurlyeq \mathcal{M}_{1}$ iff $f_{\rho}^{-1} \circ \Phi$ is an ext.-base-isomorphism.

\subsection{The case with equality}

In this subsection, let $\mathcal{A}$ be a locally finite dimensional cylindric algebra with a 
countable universe. Recall from universal algebra that an algebra $\mathcal{B}$ is a (generalized) reduct of $\mathcal{A}$ iff their universes coincide and the basic operations of $\mathcal{B}$ are term functions of $\mathcal{A}$.

Suppose $\mathcal{F} \in \mathcal{H}(\mathcal{A})$. Since $\mathcal{A}$ has a quasi-polyadic algebra reduct,

$$
\operatorname{rep}_{\mathcal{F}}: x \mapsto\left\{\tau: s_{\tau}^{+} x \in \mathcal{F}\right\}
$$

is a quasi-polyadic homomorphism onto some locally finite dimensional $\mathcal{B} \in \mathrm{Qs}_{\omega}^{R e g}$ with base $\omega$, by Lemma 3.1. Below, we show that if $\mathcal{F}$ satisfies an additional condition, namely that $d_{i j} \in \mathcal{F}$ iff $i=j$ (iff $d_{i j}=1$ ), then $\operatorname{rep}_{\mathcal{F}}$ will be a cylindric homomorphism (and $\mathcal{B}$ will be a cylindric set algebra). Let $\mathcal{H}^{\prime}(\mathcal{A})$ be the set of such ultrafilters:

$$
\mathcal{H}^{\prime}(\mathcal{A})=\mathcal{H}(\mathcal{A})-\bigcup_{i \neq j \in \omega} N_{d_{i j}}=\mathcal{H}(\mathcal{A}) \cap \bigcap_{i \neq j \in \omega} N_{-d_{i j}}
$$

These results can be considered cylindric algebraic analogs of Lemmas 3.1 and 3.2.

Lemma 4.2 If $\mathcal{F} \in \mathcal{H}^{\prime}(\mathcal{A})$, then $\operatorname{rep}_{\mathcal{F}}$ is a homomorphism from $\mathcal{A}$ onto some $\operatorname{Lf}_{\omega} \cap$ $\mathrm{Cs}_{\omega}^{\text {Reg }}$ with base $\omega$. In addition, $\operatorname{rep}_{\mathcal{F}_{0}}=\operatorname{rep}_{\mathcal{F}_{1}}$ iff $\mathcal{F}_{0}=\mathcal{F}_{1}$ for all $\mathcal{F}_{0}, \mathcal{F}_{1} \in \mathcal{H}^{\prime}(\mathcal{A})$.

Proof. Due to Lemma 3.1, all that remains to be seen is that $\operatorname{rep}_{\mathcal{F}}$ preserves $d_{i j}$ for all $i, j \in \omega$. This is so, because

$$
\begin{aligned}
\tau \in \operatorname{rep}_{\mathcal{F}}\left(d_{i j}\right) & \text { iff } \mathcal{F} \ni s_{\tau}^{+} d_{i j} \stackrel{2.3}{=} d_{\tau(i) \tau(j)} \\
& \text { iff } \tau(i)=\tau(j) \\
& \text { iff } \tau \in D_{i j} .
\end{aligned}
$$

Hence $D_{i j} \in B$ for all $i, j<\omega$, meaning that $\mathcal{B}$ is also a - regular and locally finite dimensional - cylindric set algebra.

Lemma 4.3 Suppose $\mathcal{B} \in \mathrm{Lf}_{\omega} \cap \mathrm{Cs}_{\omega}^{\text {Reg }}$ with base $\omega$, and suppose $h$ is a homomorphism from $\mathcal{A}$ to $\mathcal{B}$. Then there is an $\mathcal{F} \in \mathcal{H}^{\prime}(\mathcal{A})$ such that $h=\operatorname{rep}_{\mathcal{F}}$.

Proof. As we have seen in the proof of Lemma 3.2, for

$$
\mathcal{F}=\left\{x \in A: I d_{\omega} \in h(x)\right\}
$$

we have $\mathcal{F} \in \mathcal{H}(\mathcal{A})$ and $h=\operatorname{rep}_{\mathcal{F}}$.

Take any $i \neq j \in \omega$. Then we have $I d_{\omega} \notin D_{i j}=h\left(d_{i j}\right)$. Therefore $d_{i j} \notin \mathcal{F}$, or equivalently, $\mathcal{F} \notin N_{d_{i j}}$. Because $i$ and $j$ were chosen arbitrarily, $\mathcal{F}$ is in $\mathcal{H}^{\prime}(\mathcal{A})$. 
Remark 4.4 The construction of homomorphisms from an $\mathcal{A} \in \mathrm{Lf}_{\omega}$ with a countable universe onto $\mathrm{Lf}_{\omega} \cap \mathrm{Cs}_{\omega}^{R e g}$ 's described in [17] is not exactly the same as our construction. There, $\mathcal{H}(\mathcal{A})$ is defined as the set of those ultrafilters that preserve $\left\{s_{j}^{i} x: j \in \omega-\Delta x\right\}$ for all $x \in A$ and $i \in \omega$ (instead of preserving $\left\{s_{j}^{i} x: j \in \omega\right\}$ ). Given an arbitrary $\mathcal{F} \in \mathcal{H}(\mathcal{A})$, an equivalence relation $E=\left\{\langle i, j\rangle: d_{i j} \in \mathcal{F}\right\}$ is defined in [17] and is called the kernel of $\mathcal{F}$. Using this $E, \operatorname{rep}_{\mathcal{F}}(x)$ is defined there as

$$
\operatorname{rep}_{\mathcal{F}}(x)=\left\{\tau / E: s_{\tau}^{+} x \in F\right\} .
$$

It is plain to see that if we ignore the differnce in the definitions of $\mathcal{H}(\mathcal{A})$, then $\mathcal{H}^{\prime}(\mathcal{A})$ is the set of ultrafilters of $\mathcal{H}(\mathcal{A})$ whose kernel is the identity relation, and that for these ultrafilters, the definition of $\operatorname{rep}_{\mathcal{F}}$ in [17] coincides with our definition.

Let $\mathcal{A}=\mathrm{CA}(\Sigma)$ for some theory $\Sigma$ in a language $L$ with equality. Due to Lemmas 4.2 and 4.3 , there is a one-one correspondence between $K_{\Sigma}$ and $\mathcal{H}^{\prime}(\mathcal{A})$, just as in the case without equality. The next theorem tells us that ultrafilters of $\mathcal{H}^{\prime}(\mathcal{A})$ that lead to pairwise non elementarily embeddable models are characterized exactly as in the case without equality (see Theorem 4.1). Its proof is no different from that of Theorem 4.1.

Theorem 4.5 Let $\mathcal{M}_{0}$ and $\mathcal{M}_{1}$ be models in $K_{\Sigma}$, and let $\mathcal{F}_{0}, \mathcal{F}_{1} \in \mathcal{H}^{\prime}(\mathcal{A})$. Suppose $\operatorname{rep}_{\mathcal{F}_{i}}$ is a homomorphism from $\mathcal{A}$ onto $\operatorname{Cs}_{\omega}\left(\mathcal{M}_{i}\right)(i=0,1)$. Let $\rho: \omega \longrightarrow \omega$ be injective, and let $U=$ Range $\rho$. For each $X \subseteq{ }^{\omega} \omega$, define $f_{\rho}(X)=\{\rho \circ \sigma: \sigma \in X\}$, and for each $X \in \mathrm{Cs}_{\omega}\left(\mathcal{M}_{1}\right)$ let $\Phi(X)=X \cap{ }^{\omega} U$. Then the following are equivalent:

1. $\rho: \mathcal{M}_{0} \preccurlyeq \mathcal{M}_{1}$,

2. $f_{\rho} \circ \operatorname{rep}_{\mathcal{F}_{0}}=\Phi \circ \operatorname{rep}_{\mathcal{F}_{1}}$,

3. $\mathcal{F}_{0}=\left(s_{\rho}^{+}\right)^{-1} \mathcal{F}_{1}$.

\section{Vaught's conjecture and its variants}

In this section, we prove our main results. Here, we will treat the case with equality and the case without it simultaniously. We therefore suppose $\Sigma$ is a theory in a countable language $L$ either with or without the equality symbol. When studying Vaught's conjecture, it is usually assumed that $\Sigma$ is a complete theory. We do not assume this however, because we will not need it in proving our results. We set $\mathcal{A}=\mathrm{QPA}(\Sigma)$ and $\mathcal{H}=\mathcal{H}(\mathcal{A})$ if $L$ does not contain the equality symbol. In the case with equality, we set $\mathcal{A}=\mathrm{CA}(\Sigma)$, and $\mathcal{H}=\mathcal{H}^{\prime}(\mathcal{A})$. Below, a definition from Section 1 is recalled and made explicit.

Definition 5.1 Let $\operatorname{Inj}(\omega)$ denote the set of injective functions of ${ }^{\omega} \omega$. Given a submonoid $S$ of $\operatorname{Inj}(\omega)$, we define 
$I(\Sigma, S)=\sup \left\{|M|: M \subseteq K_{\Sigma}\right.$ and no $\rho \in S$ is an elementary embedding between any two models in $M\}$.

By Theorem 4.1 and its counterpart Theorem 4.5, we have

$$
I(\Sigma, S)=\sup \{|\mathcal{R}|: \mathcal{R} \subseteq \mathcal{H} \text { and }
$$

for every $\rho \in S$ and $\mathcal{F}_{0}, \mathcal{F}_{1} \in \mathcal{R}$ we have $\left.\mathcal{F}_{0} \neq\left(s_{\rho}^{+}\right)^{-1} \mathcal{F}_{1}\right\}$.

As pointed out in Section 1, the variant of Vaught's conjecture concerning elementary embeddings can be rephrased as follows:

$$
\text { if } I(\Sigma, \operatorname{Inj}(\omega))>\aleph_{0} \text {, then } I(\Sigma, \operatorname{Inj}(\omega))=2^{\aleph_{0}} .
$$

Finally, we recall that we endowed $\omega$ with the discrete topology and ${ }^{\omega} \omega$ with the resulting product topology. Our goal in this section is to prove that $I(\Sigma, S)>\aleph_{0}$ implies $I(\Sigma, S)=2^{\aleph_{0}}$ for any $\sigma$-compact submonoid $S$ of $\operatorname{Inj}(\omega)$ (see Theorems 5.8 and 5.9 below). A subset $X \subseteq{ }^{\omega} \omega$ is defined to be $\sigma$-compact iff there exists a countable family $\left\{X_{i} \subseteq{ }^{\omega} \omega: i<\omega\right\}$ of compact subsets of ${ }^{\omega} \omega$ such that $X=$ $\bigcup_{i<\omega} X_{i}$.

When stating Lemmas 5.2 to 5.7, we assume $S \subseteq \operatorname{Inj}(\omega)$ is a fixed $\sigma$-compact monoid, and we let $\mathcal{R}_{\Sigma}$ denote a fixed subset of $\mathcal{H}$ such that for every $\mathcal{F}_{0}, \mathcal{F}_{1} \in \mathcal{R}_{\Sigma}$ and every $\rho \in S$ we have $\mathcal{F}_{0} \neq\left(s_{\rho}^{+}\right)^{-1} \mathcal{F}_{1}$. Lemmas 5.2 to 5.6 were adapted from an earlier version of Section 3 of [17]; they are the technical cornerstones of our construction.

Lemma 5.2 Suppose $N \subseteq \mathcal{U}(\mathcal{A})$ is a clopen set of $\mathcal{A}^{*}$ such that $\left|N \cap \mathcal{R}_{\Sigma}\right| \geq \aleph_{1}$. For every $x \in A$ and every $i<\omega$ there exists a clopen $N^{\prime} \subseteq N$ such that $N^{\prime} \subseteq \mathcal{U}_{i, x}(\mathcal{A})$ and $\left|N^{\prime} \cap \mathcal{R}_{\Sigma}\right| \geq \aleph_{1}$.

Proof. For each $j \in \omega$ let $M_{j}=\left\{\mathcal{F} \in N \cap \mathcal{R}_{\Sigma}: s_{j}^{i} x \in \mathcal{F}\right\}$ and let $M_{-1}=\{\mathcal{F} \in$ $\left.N \cap \mathcal{R}_{\Sigma}: c_{i} x \notin \mathcal{F}\right\}$. Since $\mathcal{R}_{\Sigma} \subseteq \mathcal{H}$, it follows that

$$
\text { (*) } \quad N \cap \mathcal{R}_{\Sigma}=\bigcup_{j \in\{-1\} \cup \omega} M_{j} .
$$

Since the cardinality of the left hand side of $(*)$ is at least $\aleph_{1}$, it follows that there is a $j \in\{-1\} \cup \omega$ for which $\left|M_{j}\right| \geq \aleph_{1}$. If $j=-1$, then let $N^{\prime}=N \cap N_{-c_{i} x}=\{\mathcal{F} \in$ $\left.N:-c_{i} x \in \mathcal{F}\right\}$; if $j \in \omega$, then let $N^{\prime}=N \cap N_{s_{j}^{i} x}=\left\{\mathcal{F} \in N: s_{j}^{i} x \in \mathcal{F}\right\}$. In both cases, $N^{\prime}$ satisfies the requirements of the lemma.

Lemma 5.3 Suppose $N$ is a clopen set of $\mathcal{A}^{*}$ and $\left|N \cap \mathcal{R}_{\Sigma}\right| \geq \aleph_{1}$. Then there exist disjoint clopen sets $N^{\prime}, N^{\prime \prime} \subseteq N$ such that $\left|N^{\prime} \cap \mathcal{R}_{\Sigma}\right| \geq \aleph_{1}$ and $\left|N^{\prime \prime} \cap \mathcal{R}_{\Sigma}\right| \geq \aleph_{1}$. 
Proof. Suppose, seeking a contradiction that there does not exist suitable $N^{\prime}$ and $N^{\prime \prime}$. It follows that if $N^{\prime} \subseteq N$ is clopen with $\left|N^{\prime} \cap \mathcal{R}_{\Sigma}\right| \geq \aleph_{1}$, then for any clopen set $M$ either

(a) $\left|N^{\prime} \cap \mathcal{R}_{\Sigma} \cap M\right| \geq \aleph_{1}$ and $\left|\left(N^{\prime} \cap \mathcal{R}_{\Sigma}\right)-M\right|<\aleph_{1}$ or

(b) $\left|\left(N^{\prime} \cap \mathcal{R}_{\Sigma}\right)-M\right| \geq \aleph_{1}$ and $\left|N^{\prime} \cap \mathcal{R}_{\Sigma} \cap M\right|<\aleph_{1}$.

Let $\left\langle a_{i}: i \in \omega\right\rangle$ be an enumeration of the elements of $\mathcal{A}$. Let $N_{0}=N$. By recursion, we will construct a decreasing sequence $\left\langle N_{i}: i \in \omega\right\rangle$ of clopen sets such that for every $i \in \omega$ we have

$$
\text { (i) }\left|N_{i} \cap \mathcal{R}_{\Sigma}\right| \geq \aleph_{1} \quad \text { and } \quad(i i) N_{a_{i-1}} \cap N_{i} \neq \emptyset \text { implies } N_{i} \subseteq N_{a_{i-1}} \text {. }
$$

Suppose $i<\omega$ and $N_{j}$ have been defined for every $j<i$ such that $(i),(i i)$ hold. If $\left|N_{i-1} \cap \mathcal{R}_{\Sigma} \cap N_{a_{i-1}}\right| \geq \aleph_{1}$, then let $N_{i}=N_{i-1} \cap N_{a_{i-1}}$; otherwise let $N_{i}=N_{i-1}-N_{a_{i-1}}$. By (a) and (b) above, $N_{i}$ still satisfies $(i)$; in addition (ii) holds by construction. In this way $\left\langle N_{i}: i \in \omega\right\rangle$ can be completely built up.

Observe that for any $i \in \omega$ we have $\left|\left(N-N_{i}\right) \cap \mathcal{R}_{\Sigma}\right|<\aleph_{1}$ (otherwise by $(i), N_{i}$ and $N-N_{i}$ would witness a contradiction to our indirect assumption). Hence,

$$
H=\bigcup_{i \in \omega}\left(\left(N-N_{i}\right) \cap \mathcal{R}_{\Sigma}\right)
$$

is countable. Consequently, $\left(N \cap \mathcal{R}_{\Sigma}\right)-H$ is uncountable; particularly, there exist two different elements $\mathcal{F}, \mathcal{G} \in\left(N \cap \mathcal{R}_{\Sigma}\right)-H$. Since $\mathcal{F} \neq \mathcal{G}$, there exists $a_{i} \in A$ with $a_{i} \in \mathcal{F}-\mathcal{G}$.

Since $a_{i} \in \mathcal{F}$, it follows that $\mathcal{F} \in N_{a_{i}}$. In addition, since $\mathcal{F} \notin H$, it follows that $\mathcal{F} \in N_{i+1}$. So $\mathcal{F} \in N_{a_{i}} \cap N_{i+1} \neq \emptyset$, whence, by (ii) we have $N_{i+1} \subseteq N_{a_{i}}$. Similarly, $\mathcal{G} \notin H$ implies $\mathcal{G} \in N_{i+1}$. Hence $\mathcal{G} \in N_{a_{i}}$ and thus $a_{i} \in \mathcal{G}$, contradicting the choice of $a_{i}$.

Lemma 5.4 Suppose $S_{0} \subseteq S$ is a compact subset of ${ }^{\omega} \omega$. Then for every distinct $\mathcal{F}, \mathcal{G} \in \mathcal{R}_{\Sigma}$ there exist $a, b \in A$ such that

1. $a \in \mathcal{F}$ and $b \in \mathcal{G}$,

2. for every $\rho \in S_{0}$ we have $s_{\rho}^{+} a \leq-b$.

Proof. Suppose $\mathcal{F} \neq \mathcal{G} \in \mathcal{R}_{\Sigma}$. Then, by the definition of $\mathcal{R}_{\Sigma}$, for any $\rho \in S_{0}$ we have $\mathcal{F} \neq\left(s_{\rho}^{+}\right)^{-1} \mathcal{G}$. That is, for any $\rho \in S_{0}$ there exists an $a_{\rho} \in A$ such that $a_{\rho} \in \mathcal{F}$ iff $s_{\rho}^{+} a_{\rho} \notin \mathcal{G}$. Taking the complement of $a_{\rho}$ if necessary, we may assume $a_{\rho} \in \mathcal{F}$ and $s_{\rho}^{+} a_{\rho} \notin \mathcal{G}$.

For every $\rho \in S_{0}$, we let $U_{\rho}=\left\{\sigma \in{ }^{\omega} \omega: \sigma_{\mid \Delta a_{\rho}}=\rho_{\mid \Delta a_{\rho}}\right\}$. Because $\mathcal{A}$ is locally finite dimensional, $U_{\rho}$ is an open subset of ${ }^{\omega} \omega$. In addition, $\rho \in U_{\rho}$. Therefore 
$\left\{U_{\rho}: \rho \in S_{0}\right\}$ is an open covering of $S_{0}$. Since $S_{0}$ is compact, there exists $n<\omega$ and $\rho_{0}, \ldots \rho_{n-1} \in S_{0}$ such that $S_{0} \subseteq \bigcup_{i<n} U_{\rho_{i}}$.

Let

$$
a=\bigwedge_{i<n} a_{\rho_{i}} \text { and } b=\bigwedge_{i<n}-s_{\rho_{i}}^{+} a_{\rho_{i}} .
$$

Obviously, $a$ and $b$ satisfy 1 . To see that 2. is satisfied, note that for any $\rho \in S_{0}$ we have $\rho \in U_{\rho_{i}}$ for some $i<n$. Therefore using this $i$ we have $s_{\rho}^{+} a \leq s_{\rho}^{+} a_{\rho_{i}}=s_{\rho_{i}}^{+} a_{\rho_{i}} \leq$ $\bigvee_{j<n} s_{\rho_{j}}^{+} a_{\rho_{j}}=-b$, as desired.

Lemma 5.5 Suppose $S_{0} \subseteq S$ is a compact subset of ${ }^{\omega} \omega$. Let $N$ and $M$ be disjoint clopen sets of $\mathcal{A}^{*}$ such that $\left|N \cap \mathcal{R}_{\Sigma}\right| \geq \aleph_{1}$ and $\left|M \cap \mathcal{R}_{\Sigma}\right| \geq \aleph_{1}$. Then there exist $a, b \in A$ such that

1. $\left|N \cap N_{a} \cap \mathcal{R}_{\Sigma}\right| \geq \aleph_{1}$ and $\left|M \cap N_{b} \cap \mathcal{R}_{\Sigma}\right| \geq \aleph_{1}$,

2. for every $\rho \in S_{0}$ we have $s_{\rho}^{+} a \leq-b$.

Proof. Given any $\mathcal{F} \in N \cap \mathcal{R}_{\Sigma}$ and $\mathcal{G} \in M \cap \mathcal{R}_{\Sigma}$ we can find $a_{\mathcal{F}, \mathcal{G}}, b_{\mathcal{F}, \mathcal{G}} \in A$ such that $a_{\mathcal{F}, \mathcal{G}} \in \mathcal{F}, b_{\mathcal{F}, \mathcal{G}} \in \mathcal{G}$ and $s_{\rho}^{+} a_{\mathcal{F}, \mathcal{G}} \leq-b_{\mathcal{F}, \mathcal{G}}$ for every $\rho \in S_{0}$, by Lemma 5.4. Because $\left|M \cap \mathcal{R}_{\Sigma}\right| \geq \aleph_{1}$ and $\mathcal{A}$ has a countable universe, for any $\mathcal{F} \in N \cap \mathcal{R}_{\Sigma}$ there exist $a_{\mathcal{F}}, b_{\mathcal{F}} \in A$ such that for at least $\aleph_{1}$ many $\mathcal{G} \in M \cap \mathcal{R}_{\Sigma}$ we have both $a_{\mathcal{F}, \mathcal{G}}=a_{\mathcal{F}}$ and $b_{\mathcal{F}, \mathcal{G}}=b_{\mathcal{F}}$. For similar reasons, we can find $a, b \in A$ such that for at least $\aleph_{1}$ many $\mathcal{F} \in N \cap \mathcal{R}_{\Sigma}$ we have both $a_{\mathcal{F}}=a$ and $b_{\mathcal{F}}=b$. These $a$ and $b$ clearly satisfy the lemma.

Lemma 5.6 Suppose $S_{0} \subseteq S$ is compact. Let $2 \leq n<\omega$ and $\left\{N_{i}: i<n\right\}$ be disjoint clopen sets of $\mathcal{A}^{*}$ such that $\left|N_{i} \cap \mathcal{R}_{\Sigma}\right| \geq \aleph_{1}$ for all $i<n$. Then there exist $a_{0}, \ldots, a_{n-1} \in A$ such that

1. $\left|N_{i} \cap N_{a_{i}} \cap \mathcal{R}_{\Sigma}\right| \geq \aleph_{1}$ for all $i<n$,

2. for all $i<j<n$ there exists an $a_{i j} \in A$ such that $N_{i} \cap N_{a_{i}} \subseteq N_{a_{i j}}$ and for every $\rho \in S_{0}$ we have $N_{j} \cap N_{a_{j}} \subseteq N_{-s_{\rho}^{+} a_{i j}}$.

Proof. We prove this theorem by iterating the application of Lemma 5.5. In more detail, first suppose $n=2$. Then, due to Lemma 5.5, there exist $a, b \in A$ such that $\left|N_{0} \cap N_{a} \cap \mathcal{R}_{\Sigma}\right| \geq \aleph_{1}$ and $\left|N_{1} \cap N_{b} \cap \mathcal{R}_{\Sigma}\right| \geq \aleph_{1}$, and for every $\rho \in S_{0}$ we have $s_{\rho}^{+} a \leq-b$. Then $a_{0}=a, a_{1}=b$ and $a_{01}=a$ clearly satisfy 1 . and 2 .

Now, suppose $n>2$. Let $W=\{\langle i, j\rangle: i<j<n\}$. We will show that the following holds for every $J \subseteq \mathcal{P}(W)$, by induction on $|J|$ :

for every $i<n$ there exists $a_{i} \in A$ such that $\left|N_{i} \cap N_{a_{i}} \cap \mathcal{R}_{\Sigma}\right| \geq \aleph_{1}$, and if $\langle i, j\rangle \in J$, then there exists an $a_{i j} \in A$ such that $N_{i} \cap N_{a_{i}} \subseteq N_{a_{i j}}$ and for every $\rho \in S_{0}$ we have $N_{j} \cap N_{a_{j}} \subseteq N_{-s_{\rho}^{+} a_{i j}}$. 
This is enough, because $J=W$ implies the conclusion of the lemma. First, if $J=\emptyset$, then $a_{0}=a_{1}=\cdots=a_{n-1}=1$ is suitable. Now, suppose $|J|=k$ and $(*)$ holds for all $J^{\prime} \subseteq W$ such that $\left|J^{\prime}\right|<k$. Take an arbitrary $\langle i, j\rangle \in J$, and let $J^{\prime}=J-\{\langle i, j\rangle\}$. Then $\left|J^{\prime}\right|=k-1$; consequently, there exist $c_{0}, \ldots, c_{n-1}$ such that for every $l<n$ we have $\left|N_{l} \cap N_{c_{l}} \cap \mathcal{R}_{\Sigma}\right| \geq \aleph_{1}$, and if $\langle l, m\rangle \in J$, then there exists an $a_{l m}$ such that $N_{l} \cap N_{c_{l}} \subseteq N_{a_{l m}}$ and for every $\rho \in S_{0}$ we have $N_{m} \cap N_{c_{m}} \subseteq N_{-s_{\rho}^{+} a_{l m}}$. If $l \neq i, j$, then let $a_{l}=c_{l}$. Applying the case $n=2$ to $N_{i} \cap N_{c_{i}}$ and $N_{j} \cap N_{c_{j}}$, there exist $d_{i}, d_{j} \in A$ such that both

$$
\left|N_{i} \cap N_{c_{i}} \cap N_{d_{i}} \cap \mathcal{R}_{\Sigma}\right| \geq \aleph_{1} \text { and }\left|N_{j} \cap N_{c_{j}} \cap N_{d_{j}} \cap \mathcal{R}_{\Sigma}\right| \geq \aleph_{1}
$$

Let $a_{i}=c_{i} \wedge d_{i}$ and $a_{j}=c_{j} \wedge d_{j}$. This choice of $a_{l}$ and $a_{l m}$ (for all $l, m<n$ ) satisfies $(*)$ for $J$, hence the induction is complete.

Lemma 5.7 Suppose $S_{0} \subseteq S$ is compact. Let $2 \leq n<\omega$ and $\left\{N_{i}: i \in n\right\}$ be disjoint clopen sets of $\mathcal{A}^{*}$ such that $\left|N_{i} \cap \mathcal{R}_{\Sigma}\right| \geq \aleph_{1}$ for all $i \in n$. Then there exist $N_{i}^{\prime} \subseteq N_{i}$ such that

1. $\left|N_{i}^{\prime} \cap \mathcal{R}_{\Sigma}\right| \geq \aleph_{1}$ for all $i \in n$,

2. for all $i \neq j \in n$ there exists an $a_{i j}^{\prime} \in A$ such that $N_{i}^{\prime} \subseteq N_{a_{i j}^{\prime}}$ and for every $\rho \in S_{0}$ we have $N_{j}^{\prime} \subseteq N_{-s_{\rho}^{+} a_{i j}^{\prime}}$.

Proof. We prove this lemma by applying the previous one twice. In more detail, if $N_{i}$ are as in the lemma, then, by Lemma 5.6, there exist $a_{0}, \ldots, a_{n-1} \in A$ such that $\left|N_{i} \cap N_{a_{i}} \cap \mathcal{R}_{\Sigma}\right| \geq \aleph_{1}$ for all $i<n$, and for all $i<j<n$ there exist $a_{i j} \in A$ such that $N_{i} \cap N_{a_{i}} \subseteq N_{a_{i j}}$ and $N_{j} \cap N_{a_{j}} \subseteq N_{-s_{\rho}^{+} a_{i j}}$ for all $\rho \in S_{0}$.

For each $i<n$, let $M_{i}=N_{n-(i+1)} \cap N_{a_{n-(i+1)}}$. Again by Lemma 5.6, there exist $b_{0}, \ldots b_{n-1} \in A$ such that $\left|M_{i} \cap N_{b_{i}}\right| \geq \aleph_{1}$, and for all $i<j<n$ there exist $b_{i j} \in A$ such that $M_{i} \cap N_{b_{i}} \subseteq N_{b_{i j}}$ and $M_{j} \cap N_{b_{j}} \subseteq N_{-s_{\rho}^{+} b_{i j}}$ for all $\rho \in S_{0}$.

For each $i<n$, let $N_{i}^{\prime}=M_{n-(i+1)} \cap N_{b_{n-(i+1)}}$. If $i<j$, then let $a_{i j}^{\prime}=a_{i j}$, and if $j<i$, then let $a_{i j}^{\prime}=b_{n-(i+1), n-(j+1)}$. This choice of $N_{i}$ and $a_{i j}^{\prime}$ satisfies the conclusion of the lemma.

We are now ready to prove the main results of the paper.

Theorem 5.8 (Main theorem of the paper for the case without equality.) Let $\Sigma$ be a theory in a countable language without equality, and let $S \subseteq \operatorname{Inj}(\omega)$ be a $\sigma$-compact monoid.

$$
\text { If } I(\Sigma, S)>\aleph_{0} \text {, then } I(\Sigma, S)=2^{\aleph_{0}} \text {. }
$$


Proof. The idea behind this proof is the same as the one behind the proof of Theorem 3.6 of [17]. Let $\mathcal{A}=\operatorname{QPA}(\Sigma)$ and $\mathcal{H}=\mathcal{H}(\mathcal{A})$. Suppose that for $\mathcal{R}_{\Sigma} \subseteq \mathcal{H}$ we have $\left|\mathcal{R}_{\Sigma}\right|>\aleph_{0}$ and for every $\mathcal{F}_{0}, \mathcal{F}_{1} \in \mathcal{R}_{\Sigma}$ and every $\rho \in S$ we have $\mathcal{F}_{0} \neq\left(s_{\rho}^{+}\right)^{-1} \mathcal{F}_{1}$.

Suppose $\left\{S_{i} \subseteq{ }^{\omega} \omega: i<\omega\right\}$ is a countable collection of compact subsets of $\omega_{\omega}$ such that $S=\bigcup_{i<\omega} S_{i}$, and let $r=\left\langle r_{i}: i<\omega\right\rangle$ be an enumeration of $\omega$ in which for each $j<\omega$ we have $r_{i}=j$ infinitely many times. Furthermore, let $s=\left\langle s_{i}: i<\omega\right\rangle$ be an enumeration of $\{\langle i, x\rangle: i<\omega, x \in A\}$.

We show that a tree $\left\{t_{p}: p \in{ }^{<\omega} 2\right\}$ can be constructed such that the following hold for all $p, q \in{ }^{<\omega} 2$ :

1. $t_{p}$ is a clopen set of $\mathcal{A}^{*}$,

2. $\left|t_{p} \cap \mathcal{R}_{\Sigma}\right| \geq \aleph_{1}$,

3. if $p \subseteq q$, then $t_{p} \supseteq t_{q}$,

4. $t_{p \frown 0}$ and $t_{p \frown 1}$ are disjoint,

5. $t_{p} \subseteq \mathcal{U}_{s_{|p|-1}}(\mathcal{A})$

6. if $|p|=|q|$ and $p \neq q$, then there is an $a \in A$ such that for every $\rho \in S_{r_{|p|-1}}$ we have $t_{p} \subseteq N_{a}$ and $t_{q} \subseteq N_{-s_{\rho}^{+} a}$.

Let $t_{\langle\rangle}=\mathcal{U}(\mathcal{A})$. Now suppose $t_{p}$ has been defined for all $p \in{ }^{i-1} 2$ so that 1.-6. hold. Due to Lemma 5.3, for any $p \in{ }^{<i} 2$ there exist $z_{p}-0, z_{p-1} \subseteq t_{p}$ that are disjoint clopen subsets of $t_{p}$ such that $\left|z_{p} \frown \cap \mathcal{R}_{\Sigma}\right| \geq \aleph_{1}$ for $i=0,1$. Next, by Lemma 5.7, we can find for all $p \in{ }^{i} 2$ a $z_{p}^{\prime} \subseteq z_{p}$ such that the following (i) and (ii) are satisfied:

(i) $\left|z_{p}^{\prime} \cap \mathcal{R}_{\Sigma}\right| \geq \aleph_{1}$, and

(ii) if $p \neq q$, then there exists an $a \in A$ such that $z_{p}^{\prime} \subseteq N_{a}$ and $z_{q}^{\prime} \subseteq N_{-s_{\rho}^{+} a}$ for all $\rho \in S_{r_{|p|-1}}$.

Lastly, by Lemma 5.2, for all $p \in{ }^{i} 2$ there exist $t_{p} \subseteq z_{p}^{\prime}$ such that $t_{p} \subseteq \mathcal{U}_{s_{|p|-1}}(\mathcal{A})$ and $\left|t_{p} \cap \mathcal{R}_{\Sigma}\right| \geq \aleph_{1}$. It is easy to see that these $t_{p}$ 's satisfy 1.-6.

Thus a tree $\left\langle t_{p}: p \in{ }^{<i} 2\right\rangle$ can be constructed so that statements 1.-6. are satisfied.

Take any $f \in \omega^{\omega} 2$. Then $\left\langle t_{f_{\mid n}}: n<\omega\right\rangle$ is a decreasing sequence of nonempty clopen sets of $\mathcal{A}^{*}$ by 1., 2., and 3., and therefore it has the finite intersection property. Because $\mathcal{A}^{*}$ is a compact space, this implies that $\bigcap_{i<\omega} t_{f_{\mid n}} \neq \emptyset$. For every $f \in{ }^{\omega} 2$, take an arbitrary $\mathcal{F}(f) \in \bigcap_{i<\omega} t_{f_{\mid n}}$. By 5 ., we have $\mathcal{F}(f) \in \mathcal{H}$.

Suppose $f \neq g$ and for some $\rho \in S$ we have

$$
\mathcal{F}(f)=\left(s_{\rho}^{+}\right)^{-1} \mathcal{F}(g) .
$$

Let $n<\omega$ be such that $f(n) \neq g(n)$. Then for some $i>n$ we have $\rho \in S_{r_{i-1}}$. By construction, $t_{f_{\mid i}} \neq t_{g_{\mid i}}$, so 6 . implies that for some $a \in A$ we have $\mathcal{F}(f) \in t_{f_{\mid i}} \subseteq N_{a}$ and also $\mathcal{F}(g) \in t_{g_{\mid i}} \subseteq N_{-s_{\rho}^{+} a}$. That is, $a \in \mathcal{F}(f)$ and $-s_{\rho}^{+} a \in \mathcal{F}(g)$. But because of $(*)$, we also have $s_{\rho}^{+} a \in \mathcal{F}(g)$, an obvious contradiction.

Thus $\mathcal{H}$ contains $2^{\aleph_{0}}$ many ultrafilters corresponding to pairwise non $S$-elementarily embeddable models, and so $I(\Sigma, S)=2^{\aleph_{0}}$. 
Theorem 5.9 (Main theorem of the paper for the case with equality.)

Suppose $\Sigma$ is a theory in a countable language containing the equality symbol, and let $S \subseteq \operatorname{Inj}(\omega)$ be a $\sigma$-compact monoid.

$$
\text { If } I(\Sigma, S)>\aleph_{0} \text {, then } I(\Sigma, S)=2^{\aleph_{0}} \text {. }
$$

Proof. This proof differs from the previous one only in that we have to make sure that the ultrafilters obtained from the previous construction are in $\mathcal{H}^{\prime}(\mathcal{A})$; that is, for all $i \neq j \in \omega$ they are in $N_{-d_{i j}}$.

Let $\mathcal{A}=\mathrm{CA}(\Sigma)$ and $\mathcal{H}=\mathcal{H}^{\prime}(\mathcal{A})$. Suppose that for $\mathcal{R}_{\Sigma} \subseteq \mathcal{H}$ we have $\left|\mathcal{R}_{\Sigma}\right|>\aleph_{0}$ and for every $\mathcal{F}_{0}, \mathcal{F}_{1} \in \mathcal{R}_{\Sigma}$ and every $\rho \in S$ we have $\mathcal{F}_{0} \neq\left(s_{\rho}^{+}\right)^{-1} \mathcal{F}_{1}$. Let $r, s$ and $\left\{S_{i}: i<\omega\right\}$ be as in the previous proof, and let $q=\left\langle q_{k}: k<\omega\right\rangle$ be an enumeration of $\left\{-d_{i j}: i \neq j \in \omega\right\}$.

We will show that a tree $\left\langle t_{p}: p \in{ }^{<\omega} 2\right\rangle$ can be constructed, which satisfies the stipulations 1.-6. of the previous proof, as well as the following stipulation for all $p \in{ }^{<\omega} 2$ :

$$
\text { 7. } t_{p} \subseteq N_{q_{|p|-1}} \text {. }
$$

As before, let $t_{\langle\rangle}=\mathcal{U}(\mathcal{A})$. Next suppose $\left\{t_{p}: p \in{ }^{<i} 2\right\}$ have all been defined so that 1.-7. are satisfied. Then as in the previous proof, we can define for all $p \in{ }^{i} 2$ sets $t_{p}^{\prime}$ that satisfy 1.-6. Now for any $p \in{ }^{i} 2$ let $t_{p}=t_{p}^{\prime} \cap N_{q_{|p|-1}}$. It is immediate that 7 . is satisfied, and that 1. and 3.-6. remain true. Furthermore, 2. is satisfied, because for all $i \neq j \in \omega$ we have $\mathcal{R}_{\Sigma} \subseteq \mathcal{H} \subseteq N_{-d_{i j}}$ and so $\left|t_{p} \cap \mathcal{R}_{\Sigma}\right|=\left|t_{p}^{\prime} \cap N_{q_{|p|-1}} \cap \mathcal{R}_{\Sigma}\right|=\left|t_{p}^{\prime} \cap \mathcal{R}_{\Sigma}\right| \geq \aleph_{1}$ for all $p \in{ }^{i} 2$.

Thus we can build a tree $\left\langle t_{p}: p \in \epsilon^{<\omega} 2\right\rangle$ that satisfies statements 1.-7. With its help, we obtain for each $f \in{ }^{\omega} 2$ the ultrafilter $\mathcal{F}(f) \in \bigcap_{n<\omega} t_{f_{\mid n}}$ as in the proof above. $\mathcal{F}(f) \in \mathcal{H}(\mathcal{A})$ and different $f, g \in{ }^{\omega} 2$ lead ultrafilters corresponding to pairwise non $S$-elementarily embeddable models for the same reasons as in the previous proof. Furthermore, by 7 ., $\mathcal{F}(f) \in \mathcal{H}^{\prime}(\mathcal{A})=\mathcal{H}$. Thus $I(\Sigma, S)=2^{\aleph_{0}}$.

We conclude this section by a corollary concerning pairwise non-isomorphic countable models of $\Sigma$.

Corollary 5.10 Let $\Sigma$ be a theory either in a language with or without equality. Let $S \subseteq{ }^{\omega} \omega$ be a $\sigma$-compact group. Then

$$
I(\Sigma, S)>\aleph_{0} \text { implies } I(\Sigma, S)=2^{\aleph_{0}}
$$

In more detail: suppose there exists a set $\left\{\mathcal{A}_{i}: i<\aleph_{1}\right\}$ of countable models of $\Sigma$ such that for each $i<\aleph_{1}$, the universe of $\mathcal{A}_{i}$ is $\omega$, moreover, if $i \neq j<\aleph_{1}$, then for any $\varrho \in S, \varrho$ is not an isomorphism between $\mathcal{A}_{i}$ and $\mathcal{A}_{j}$ (that is, $S$ has at least $\aleph_{1}$ many orbits on models of $\Sigma$ whose universe is $\left.\omega\right)$. Then $\Sigma$ has continuum many such models (i.e., $S$ has $2^{\aleph_{0}}$ many orbits on models of $\Sigma$ whose universe is $\omega$ ). 
Proof. Let $\mathcal{A}, \mathcal{B}$ be two models of $\Sigma$ whose universes are $\omega$. Since $S$ is a group, its elements are permutations of $\omega$. Therefore the following are equivalent:

$\bullet \varrho \in S$ is an elementary embedding between $\mathcal{A}$ and $\mathcal{B}$;

$\bullet \varrho \in S$ is an isomorphism between $\mathcal{A}$ and $\mathcal{B}$.

Hence the corollary follows from Theorem 5.8 and Theorem 5.9.

\section{Concluding remarks}

In this section, we present some further questions and research directions which remain open. We believe that the methods of this paper may be further developed to handle them.

Let $\Sigma$ be a first order theory. When one investgates the number of nonisomorphic models of $\Sigma$, one usually assumes that $\Sigma$ is complete. This is because distinguishing models of $\Sigma$ by first order formulas is well understood and "easy" in some sense. We note, however, that in the present paper we did not use that $\Sigma$ is complete (particularly, the proofs of Sections 4 and 5 work for noncomplete theories as well).

Suppose $\Sigma$ is not a complete theory. Instead of taking countably infinite models of $\Sigma$, one can then take finite models as well. It would also be interesting to say something about the number of pairwise non-isomorphic models of $\Sigma$ of a fixed finite cardinality. Our methods seem to be applicable here as well. For completeness, we mention that with a completely different technique we have already started to study some extensions of Morley's categoricity theorem to the finite: if $\Sigma$ is $\aleph_{1}$-categorical (but not necessarily $\aleph_{0}$-categorical), then, under some further technical conditions, large enough finite portions of $\Sigma$ have unique $n$-element models for any large enough finite $n \in \omega$. For more details we refer to [18].

Turning back to the original problem, it would be intriguing to know whether or not the $\sigma$-compactness of $S$ may be removed from the conditions of Theorems 5.8 and 5.9. This question seems to be difficult, because, similarly to the proof of Corollary 5.10, an affirmative answer would imply Vaught's original conjecture.

An elementary mapping is a fuction that preserves all $\emptyset$-definable relations. In the context of languages without equality, an elementary mapping is not necessarily injective. By an elementary function between the structures $\mathcal{A}$ and $\mathcal{B}$, we mean an elementary mapping whose domain is the universe of $\mathcal{A}$. We can consider models up to elementary functions instead of elementary embeddings. It would be interesting to know if our methods may be adapted to this case; (we believe that this can be done).

We believe that the approach presented in this paper can be further developed to study the number of non-isomorphic $\kappa$ sized models of a theory (where $\kappa$ is a fixed, possibly uncountable cardinal). In fact, related investigations on Vaught's conjec- 
ture based on algebraic logic may also be found in [19]. There, among other results, we give a cylindric algebraic proof of Morley's celebrated theorem: $I\left(\Sigma, \aleph_{0}\right)>\aleph_{1}$ implies $I\left(\Sigma, \aleph_{0}\right)=2^{\aleph_{0}}$. In addition, using dimension complemented cylindric algebras instead of locally finite dimensonal ones, we extend our approach to certain first order logics with infinitary predicates (which is beyond the usual first order logic).

\section{References}

[1] H. Andréka, I. NÉmeti, A Simple, Purely Algebraic Proof of the Completeness of Some First Order Logics, Algebra Universalis, 5(1975) 8-15.

[2] H. Becker, A. Kechris, The Descriptive Set Theory of Polish Group Actions, Cambridge University Press, Cambridge (1996).

[3] E. Bouscaren, D. Lascar, Countable Models of Non-Multidimensional $\aleph_{0^{-}}$ stable Theories, Journal of Symbolic Logic, 48(1983) 197-205.

[4] S. Buechler, Vaught's Conjecture for Superstable Theories of Finite Rank, Ann. Pure Appl. Logic 155 (2008) No 3. 135-172.

[5] S. Buechler, Essential Stability Theory, Springer, (1996).

[6] C. C. Chang, H. J. Keisler, Model Theory, North-Holland, Amsterdam (1973).

[7] M. FERENCZI, On representability of neatly embeddable cylindric algebras, J. Appl. Non-Classical Logics, 10, 3-4, (2000), 303-315.

[8] M. Ferenczi, G. SÁgi, On Some Developments in the Representation Theory of Cylindric-Like Algebras, Algebra Universalis, Vol. 55 (2006) 345-353.

[9] L. Henkin, J. D. Monk, A. Tarski, Cylindric Algebras Part 1, NorthHolland, Amsterdam (1971).

[10] L. Henkin, J. D. Monk, A. Tarski, Cylindric Algebras Part 2, NorthHolland, Amsterdam (1985).

[11] W. Hodges, Model theory, Cambridge University Press, (1997).

[12] D. Marker, Model theory: An Introduction, Springer, (2002).

[13] M. D. Morley, The Number of Countable Models, Journal of Symbolic Logic, $35(1970)$ 14-18.

[14] I. NÉmeti, G. SÁgi, On the Equational Theory of Representable Polyadic Equality Algebras, Journal of Symbolic Logic, 65(2000), No. 3, 1143-1167. 
[15] G. SÁgi, A Completeness Theorem for Higher Order Logics, Journal of Symbolic Logic, 65(2000), No. 2, 857-884.

[16] G. SÁgi, A note on algebras of substitutions, Studia Logica 72(2002), 265-284.

[17] G. SÁGI, Vaught's Conjecture from the perspective of Algebraic Logic, submitted (2011).

[18] G. SÁgi, Z. Gyenis, Upward Morley's Theorem Downward, Manuscript, submitted (2010).

[19] T. Sayed-Ahmed, G. SÁgi Vaught's Conjecture via Cylindric Algebras, Manuscript, in preparation (2011).

[20] T. Sayed Ahmed, Martin's Axiom, Omitting Types and Complete Representations in Algebraic Logic, Studia Logica 72(2002), 285-309.

[21] S. Shelah, L. Harrington, M. Makkai A Proof of Vaught's Conjecture for $\omega$-Stable Theories, Isr. J. Math. 49 (1984), 259-280.

[22] S. Shelah, Classification theory, North-Holland, Amsterdam (1990).

[23] R. Vaught, Denumerable Models of Complete Theories, in: Infinitistic Methods, pp. 303-321, Pergamon, London, (1961).

\section{BUTE}

Department of Algebra

Budapest, Egry J. u. 1

H-1111 Hungary

e-mail: sagi@math.bme.hu
Alfréd Rényi Institute of Mathematics Hungarian Academy of Sciences Budapest Pf. 127

H-1364 Hungary

e-mail: sagi@renyi.hu

Eötvös University, Faculty of Nat. Sci.

Mathematical Institute

Budapest, Pázmány P. sétány 1/C

H-1117 Hungary

e-mail: dmvszdi@gmail.com 